\title{
CORRECTION TO THE MASS OF JUPITER DERIVED \\ FROM THE MOTION OF (153) HILDA, (279) THULE, AND \\ (334) CHICAGO
}

\author{
H. SCHOLL \\ Astronomisches Rechen-Institut, Heidelberg, B.R.D. \\ (Presented at IAU Colloquium No. 9, 'The IAU System of \\ Astronomical Constants', Heidelberg, Germany, August 12-14, 1970.)
}

\begin{abstract}
An analysis of the observations of the minor planets (153) Hilda, (279) Thule and (334) Chicago yields the following values for the reciprocal mass of Jupiter: (153) Hilda 1047.378 $\pm \mathbf{0 . 0 1 9}$, (279) Thule $1047.347 \pm 0.023$, (334) Chicago $1047.325 \pm 0.010$. A possible error in the mass of Saturn that might affect these results is discussed.
\end{abstract}

\section{Introduction}

The mass of Jupiter is determined by the motion of its satellites or by perturbational effects on nearby celestial bodies (comets, minor planets, Saturn). As is well known, minor planets, whose mean motion is commensurable to that of Jupiter, are especially suitable for the determination of the mass of Jupiter because they suffer large perturbations in mean longitude. Therefore, Hill proposed a list of 12 minor planets, all of which belong to the $2 / 1$ commensurability case. Some of them were investigated in the last few years. A paper by Klepczynski shows the results (1969).

The minor planets (153) Hilda and (334) Chicago are members of the Hilda group, which represents the $3 / 2$ commensurability case. Of this group, (334) Chicago comes closest to Jupiter (Schubart, 1968) approaching it to within 1.1 AU in the year 1917. (153) Hilda comes to within 1.9 AU of Jupiter every $24 \mathrm{yr}$.

(279) Thule is the only known planet of the $4 / 3$ commensurability type. Every $36 \mathrm{yr}$ it reaches its minimum distance from Jupiter, 1.2 AU. Observations of the three minor planets were used to find a correction to the mass of Jupiter. The following table shows some observational statistics:

\begin{tabular}{llll}
\hline Minor planet & Span & $\begin{array}{l}\text { Number of } \\
\text { observations }\end{array}$ & $\begin{array}{l}\text { Number of } \\
\text { oppositions }\end{array}$ \\
\hline (153) Hilda & $(1875-1968)$ & 128 & 37 \\
(279) Thule & $(1888-1968)$ & 90 & 33 \\
(334) Chicago & $(1892-1968)$ & 176 & 51
\end{tabular}

\section{Calculations}

The $N$-Body Program of Schubart and Stumpff (1966) was the basis for the numerical 
integrations of the planetary orbits. The planetary masses and the starting values of the orbits of the major planets were taken from this paper. The mass of Mercury was added to the solar mass and the attraction of Pluto was neglected. To obtain a reference orbit for each minor planet, the elements of the three minor planets given in "Minor Planets, 1969,' were improved. Some of the observations were used for the improvement.

For each minor planet the O-C's were represented as linear functions of the corrections to its six orbital elements and to the mass of Jupiter as a seventh unknown, the first partial derivatives of these unknowns being coefficients. This system of linear equations was solved by the least squares method. The derivatives were obtained numerically from the differences between variational orbits and the orbits of reference. This method is described in detail by Zech (1968). The variational orbit for the seventh unknown was derived by changing the mass of Jupiter from Newcomb's value of 1047.355 to 1048.5 . This change has an influence on the motion of Jupiter itself. Therefore, its osculating semi-major axis was slightly changed to keep Jupiter as close as possible to its original orbit.

\section{Results}

The following table shows the correction to the mass of Jupiter in units of the mass variation and the resulting reciprocal mass of Jupiter with the corresponding mean errors:

$\begin{array}{lll}\text { (153) Hilda } & (+1.97 \pm 1.69) \times 10^{-2} & 1047.378 \pm 0.019 \\ \text { (279) Thule } & (-0.71 \pm 1.98) \times 10^{-2} & 1047.347 \pm 0.023 \\ \text { (334) Chicago } & (-2.63 \pm 0.90) \times 10^{-2} & 1047.325 \pm 0.010\end{array}$

Taking the mean errors into account, the corrections resulting from the observations of Hilda and Thule are not significant. However, the corrections based on the observations of Chicago indicate that the reciprocal mass of Jupiter is less than Newcomb's value.

In the hope of obtaining some indication of whether the reciprocal mass of Saturn is more or less than the adopted value of 3501.6, the mass of Saturn was changed in the calculations and the mean errors were taken into account. Therefore, a correction to the mass of Jupiter was computed as above but using the reciprocal masses 3493.2 and 3510.0 for Saturn, respectively. The results are shown on the next table:

\begin{tabular}{llll}
\hline Minor Planet & \multicolumn{4}{l}{ Reciprocal Mass of Saturn } \\
& 3493.2 & 3501.6 & 3510.0 \\
\hline (153) Hilda & $1047.461 \pm 0.021$ & $1047.378 \pm 0.019$ & $1047.295 \pm 0.020$ \\
(279) Thule & $1047.309 \pm 0.027$ & $1047.347 \pm 0.023$ & $1047.384 \pm 0.024$ \\
(334) Chicago & $1047.331 \pm 0.010$ & $1047.325 \pm 0.010$ & $1047.319 \pm 0.011$ \\
\hline
\end{tabular}


For all three minor planets, the correction to the mass of Jupiter is a linear function of the mass of Saturn. With increasing reciprocal mass of Saturn, the resulting values decrease for Hilda and Chicago and increase for Thule. In the case of Chicago, the value for the reciprocal mass of Jupiter is hardly affected by slight changes in the mass of Saturn. The minima of the corresponding mean errors yield in the case of Hilda and Thule a value of 3504 and in the case of Chicago a value of 3500 for the reciprocal mass of Saturn. Combining these results, the three minor planets treated in this paper give no indication of an error in the mass of Saturn.

\section{Acknowledgements}

It is a pleasure to express my thanks to Dr J. Schubart for his continuing advice and to Professor Dr W. Fricke for helpful conversations.

The computations were carried out on the IBM 360/44 of the Rechenzentrum der Universität Heidelberg and on the Siemens 2002 of the Astronomisches RechenInstitut, Heidelberg.

\section{References}

Klepczynski, W. J.: 1969, Astron. J. 74, 774.

Schubart, J.: 1968, Astron. J. 73, 99.

Schubart, J. and Stumpff, P.: 1966, Veröffentl. Astron. Rechen-Institut Heidelberg, No. 18.

Zech, G.: 1968, Veröffentl. Astron. Rechen-Institut Heidelberg, No. 21. 